\title{
A possible alternate pathway for intravascular thrombosis-Investigation of the circumstantial evidence by microfluidics
}

\author{
Siddhartha Das ${ }^{1,2 *}$, Amlan Barai $^{1}$
}

\begin{abstract}
Bacteremia resulting in sepsis and disseminated intravascular coagulation (DIC) are known for thrombosis and coagulopathy. DIC, which results in simultaneous activation and consumption of coagulation factors, could be investigated using microfluidics as a tool. Here, we propose the hypothesis that bacteria (e.g. E.coli) mediated DIC results from a collective phenomenon called "quorum acting" (QA). If our hypothesis is true, than the coagulation cascade will be activated before systemic inflammation. To check for QA we propose to perform a hemodynamic experiment where blood is controllably flown over E.coli clusters in a microfluidic device. Further, manipulation of the physical properties (flow rate mimicking condition like venous stasis) and chemical properties (hyperglycaemia as in uncontrolled diabetes mellitus) of blood could be done using microfluidic device to mimic their etiopathogenesis and to validate our proposed mechanism that quorum acting mediated DIC occurs rapidly in venous stasis and uncontrolled diabetes mellitus respectively. In light of the literature, in this hypothesis, we aimed to settle up an experimental procedure and possible mechanism for bacteremia induced disseminated intravascular coagulation via QA.
\end{abstract}

Keywords: Sepsis; DIC; Etiopathogenesis; Quorum Acting; Hypothesis

\section{Introduction}

The proposed mechanism stems from our clinical observation that there is a high morbidity and mortality rate in patients who develops sepsis and related complications in critical care units in hospitals throughout the world. Maintaining aseptic environment in hospitals represent a major challenge. Sepsis is rarely reported as the primary diagnosis and is often a complication of some other underlying diseases. As a result, the incidence, mortality, and morbidity rates of sepsis are often underestimated (1). An understanding of the mechanism of sepsis progression can help us in devising intervention and prevention strategies. At an aggregate cost of USD 20.3 billion for 1.1 million hospitalizations, sepsis was the most expensive condition seen in the U.S. hospital stays in 2011 (2).

At present, there are no well defined approaches to intercept the development and progression of sepsis and its related complication disseminated intravascular coagulation (DIC). Most often, patients does not respond to treatment and its progressive sequelae.

Our proposed mechanism focuses to understand the blood clotting mechanism which occurs in severe sepsis and then if provides encouraging results, could be used to formulate strategies to prevent its progression. As sepsis and DIC involve high morbidity and mortality, a step towards its prevention would be extremely beneficial for a large number of people.

Blood coagulation occurs by a cascade of enzymatic reactions. At the end of this cascade soluble fibrinogen is converted to insoluble fibrin (3-5). There are two pathways through which coagulation cascade occurs, namely, extrinsic and intrinsic pathways. In this aspect we would like to focus on both intrinsic and extrinsic pathways, as both are relevant for intravascular coagulation. The serum enzyme that initiates the intrinsic cascade is coagulation factor 12 (Hageman Factor).

As shown in figure 1, the enzymatic process then propagates further and activates other coagulation factors resulting in the formation of blood clots. Though the intrinsic pathway is initiated by factor 12 (Hageman Factor), investigation could to be done to examine whether coagulation cascade can also be initiated by factors $11,9,10$ or 2 . Similar analogy could also be followed in case of the extrinsic pathway which is initiated by tissue factor or factor 3 . 


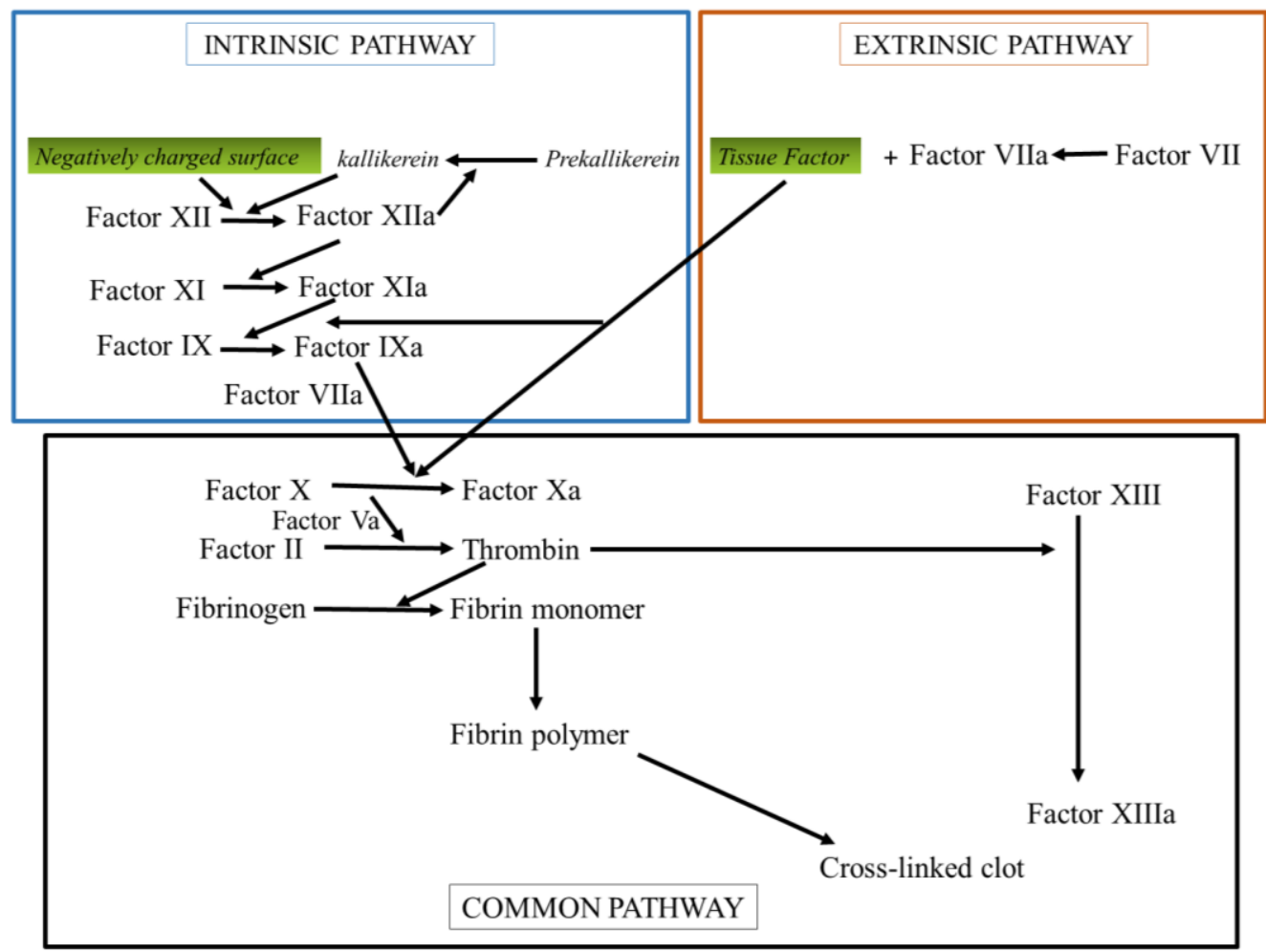

Figure 1: A schematic demonstration of classic extrinsic and intrinsic coagulation pathways. For the sake of simplicity, calcium ion, phospholipids, kininogen etc. has been omitted from the figure. Figure adapted from the literature (4)

Studies could be carried out to probe whether extrinsic pathway uses tissue factor/factor 3 for initiation of coagulation processes by a recently discovered phenomena called "quorum acting" (our proposed mechanism) or by passes and directly uses factor 7 .i.e. proconvertin to form clots. Extrinsic pathway involves factor 3 , i.e. tissue factor which arises from tissue injury. This transmembrane glycoprotein called tissue factor (TF) is present on the surface of many cell types like endothelial cells, macrophages, and monocytes and is not found in normal circulation. It is exposed to the circulation only after some pathology or vascular damage $(3,5)$.

Sepsisis a systemic response to infection (6) that later compromises oxygenated blood flow to various organs and may lead to multi-organ failure $(3,6-8)$.

The clinical symptoms for severe sepsis include body temperature higher than $38^{\circ} \mathrm{C}$ or lower than $36^{\circ} \mathrm{C}$, heart rate higher than $90 / \mathrm{min}$, hyperventilation evidenced by respiratory rate higher than $20 / \mathrm{min}$ or $\mathrm{Pa} . \mathrm{CO} 2$ lower than $32 \mathrm{~mm} \mathrm{Hg}$ and a white blood cell count higher than 12000 cells $/ \mu$ l or lower than $4,000 / \mu 1$ $(3,5,8-10)$.

Sepsis is caused by the immune system's response to a serious infection, most commonly bacteria (11).
E. Coli has been associated with a large number of sepsis cases. Sepsis is the leading cause of death in hospitalized patients $(12,13)$.Increased incidences of sepsis are seen in immunosuppressed, neutropenic, IV line carriers, elderly, diabetic, asplenic patients, alcoholics $\operatorname{etc}(3,7,14)$. Mortality rises with the number of organs involved $(3,7,14)$. About400,000 incidences of sepsis occur per year in the USA (12, 13).

Disseminated Intravascular Coagulation (DIC) is one of the most common complications of patients diagnosed with sepsis in critical care units $(1,3,7$, 10). DIC is an explosive and life-threatening bleeding disorder in which coagulation factors are activated and degraded simultaneously $(1,3,7,10)$. DIC is an acquired syndrome involving intravascular activation of coagulation with loss of localization arising from different causes $(3,7)$. It causes damage to the microvasculature, which, if sufficiently severe, can produce organ dysfunction $(1,3,7,14,15)$. The clinical picture involves bleeding and thrombosis (i.e. intravascular clot formation) $(1,3,7,8,14,15)$. A major proportion of DIC is seen in sepsis patients, although other causes, such as, diffuse endothelial injury, obstetric complications, etc. are also common $(3,7)$. 
DIC is estimated to be present in as many as $1 \%$ of hospitalized patients (13). DIC is not a specific illness, but is always secondary to an underlying disorder and is associated with a number of clinical conditions (3, 7). Sepsis and DIC are seen to occur in patients who are often in CCU/ICU. DIC is a progressive and fatal process. Absence of medical intervention at the right time leads to multi-organ dysfunction and certain death $(3,7,10,14,15)$.

Blood coagulation often accompanies bacterial infections and sepsis (16). Although bacterial cells sporadically activate coagulation factors, the activated factors do not necessarily undergo synchronized propagation (i.e. cascade) to form clots (17). A careful and detailed scientific approach is required to explore whether bacteria e.g. E. Coli induce the intrinsic coagulation cascade in the case of DIC via Quorum acting.
Kastrupet al (17) proposed that clusters of bacteria induce clot formation in blood by a process known as quorum acting. Quorum acting is different from the more well-known collective phenomenon of quorum sensing, where the gene expression in bacteria is changed leaving the bacterial environment unchanged $(17,18)$. In contrast to quorum sensing, quorum acting involves activation of the bacterial environment with no change in gene expression of the bacteria (17-19) (fig. 2(a)). Formation of bacterial clusters in confinement is one of the prerequisites for quorum acting. Human blood plasma coagulates on spatially localized bacteria in the absence of flow. As shown in fig. 2(b), a single bacterium or isolated patches of bacteria do not lead to quorum acting. On the other hand, when surface patches of bacteria are clustered together, the concentration of activated coagulation factors exceeds the threshold required for initiation of coagulation (top pink line in fig.2b).

- Bacteria

Diffusion of signals

Activated environment
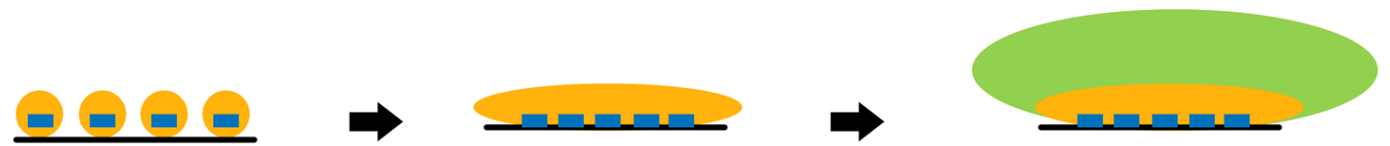

Figure 2 (a): Quorum acting .Figure adapted from (18).

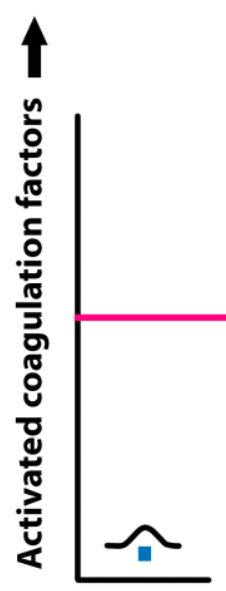

A

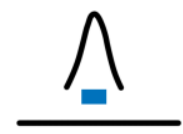

B

\section{Coagulation threshold}

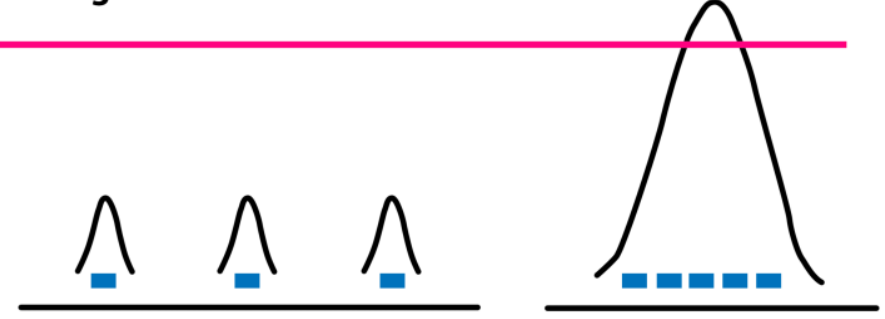

C

D

\section{Distance}

Figure 2 (b): Threshold for Quorum acting. A- Sub-threshold signal from single bacterium, B-single bacterial patch, C-separated bacterial patches, D- Clustered bacterial patches produced threshold signal for activation of coagulation. Figure adapted from the literature (18) 
The number of bacteria needs to exceed a certain threshold to initiate coagulation by quorum acting. This threshold is different for different species of bacteria. For example, 4 x103CFUs of Bacillus cereus were capable of initiating coagulation in $10 \mathrm{ml}$ of human blood plasma (17). Even though E. Coli is one of the major causes of sepsis, it is not known if (a) E. Coli initiates coagulation by quorum acting, and, (b) if so, what is its threshold number.

\section{The Proposed mechanism}

The proposed mechanism is based on the investigation of following objectives:

1) Does the coagulation factor 12 (Hageman Factor)/3(Tissue Factor) initiate coagulation cascade in presence of $E$. Coli in vitro? In case coagulation factor 12 or tissue factor is bypassed, which other factor initiates the coagulation cascade?

2) Does quorum acting mediate blood coagulation in presence of $E$. Coli clusters?

3) Does coagulation occur more rapidly in static condition (venous stasis) or under flow of blood?

4) Does hyperglycemic blood induce more rapid coagulation when compared to normoglycemic blood?

\section{Evaluation of Proposed mechanism}

We recommend the following research direction to test the mechanism of our alternate etiopathogenesis of sepsis and its complication.

\section{Research direction for objective 1}

The microfluidic device required for hemodynamic experiment for exploring objective 1 comprises of $\mathrm{Y}$ shaped micro-channels as shown in fig.3. The two inputs could be used to flow citrated blood and hydrogel droplets containing bacteria (e.g. E. Coli) respectively. Triangular pillars could be incorporated in the straight channel, as shown in the inset. The spacing $(\sim 120 \mu \mathrm{m})$ between the pillars is such that it does not let hydrogel droplets $(\sim 160 \mu \mathrm{m}$ in diameter $)$ to pass through, but allows blood to flow. As a result, there will be one or more layers of hydrogel beads stuck between these triangular pillars. The flow velocity of the blood could be adjusted such that the bacteria inside the hydrogel droplets get enough time to react with the blood and induce coagulation.

The device has to be inspected in real time using an inverted microscope. At first, the blood coming out of the device is to be examined to detect the presence of clots. Presence of clots in blood is indicated by irregular-shaped aggregates. If clots are present, blood is examined for the presence of factor $12 / 7$. If the blood that comes out of the outlet is deficient in factor $12 / 7$, it means that coagulation cascade follows the intrinsic/extrinsic pathway (i.e. uses factor $12 / 7$ to initiate it). However, if clots are present and factor $12 / 7$ is also found in the blood after clot formation, then it could be inferred that coagulation cascade follows a different pathway. Later, further tests could be carried out to quantitate the value of the individual coagulation factors in blood to understand the new pathway better. The following sections highlight the experimental steps.

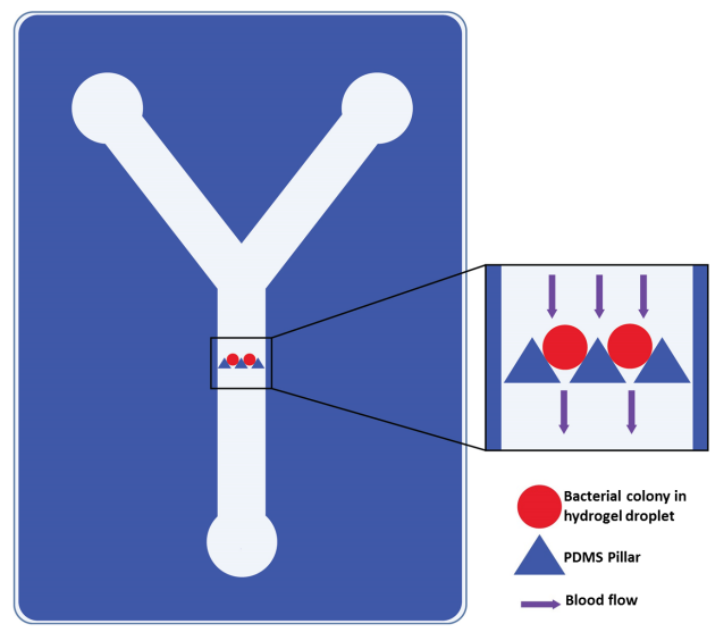

Figure 3: Microfluidic device for objective 1

(a) Device design and fabrication: Photolithography could be used to design the template for fabricating the device. Photolithography is the optical means for transferring patterns onto a substrate $(20,21)$. Patterns are first transferred from a mask to a photosensitive polymer layer (photoresist). Photoresist can be spun on a substrate and then exposed to UV radiation through a mask containing the desired pattern $(20,21)$.

"Soft lithography" could be employed for molding the actual device from the elastomer polydimethylsiloxane (PDMS) using the template thus fabricated. PDMS prepolymer and curing agent are mixed in 10:1 ratio and poured on the template and cured at $65^{\circ} \mathrm{C}$ for 45 minutes. Then the cured PDMS is peeled off from the template. Then access holes are punched in the PDMS chip to define reservoirs. The mold could be examined by SEM imaging and profilometry to check the sizes and the shapes of the triangular pillars. Next, the PDMS chip and clean glass coverslips could be irreversibly bonded using oxygen plasma to obtain the sealed microfluidic device $(20,21)(44)$.

Flow testing of the device: Once the micro device is prepared, it could be tested for different actual flow conditions using coloured liquids and/or fluorescent dyes. It is to rule out leakage or blockage of channels.

Testing of the device using blood: The Samples of citrated blood could be obtained from a bloodbank. Usually blood is mixed with sodium citrate to prevent coagulation in storage condition. It inhibits calcium ions (factor 4) which are required for propagation of the coagulation cascade $(3,7,14)$. Positively-charged calcium ions are essential to pin down the coagulation 
factors to the phospholipid so that they are closer to each other $(3,7,14)$. Coagulation factors also have negatively charged $\gamma$-carboxylated glutamic acid residue which gets bonded by calcium ions with the help of vitamin $\mathrm{K}(3,7,14)$. These processes are vital for the propagation of the coagulation cascade. In order to nullify the effect of citrate, calcium could be added in blood sample just before running the experiment. For hemodynamic-experiments, the blood sample needs to be clinically stable and free of pathogenic microorganisms and infections. Prior to experiments, the blood sample could be given to a pathological laboratory to perform basic screening and rule out contaminations and infections.

Production of agarose hydrogels containing bacteria: A suspension of $E$. Coli in agarose solution could be prepared. Since agarose is nontoxic and porous, it allows E.coli to derive nutrition from the medium as well as excrete efficiently.
This results in the formation of clustered E.coli cells of sufficient numbers when incubated at mesothermic temperature. Next, a microfluidic device having "flow-focusing" geometry could be used (fig.4) to generate agarose gel droplets containing bacteria (22). The device consists of a cross junction with three inputs and one outlet channel.

Mineral oil and E. Coli suspension in agarose could be passed through the two side inputs and the central channel respectively. As mineral oil and the cell solution are immiscible, one can generate droplets by adjusting the flow rates of the two liquids with the help of syringe pumps. (22-24).

The " $T$ "- junction geometry helps the cell solution to break into discrete droplets which are then carried by the mineral oil. Fig. 4, shows the schematic diagram of the device geometry required to generate droplets containing bacterial suspension. The channels could be filled with coloured fluid to increase the contrast. The arrow in fig. 4 shows the direction of flow of the agarose solution mixed with cells.

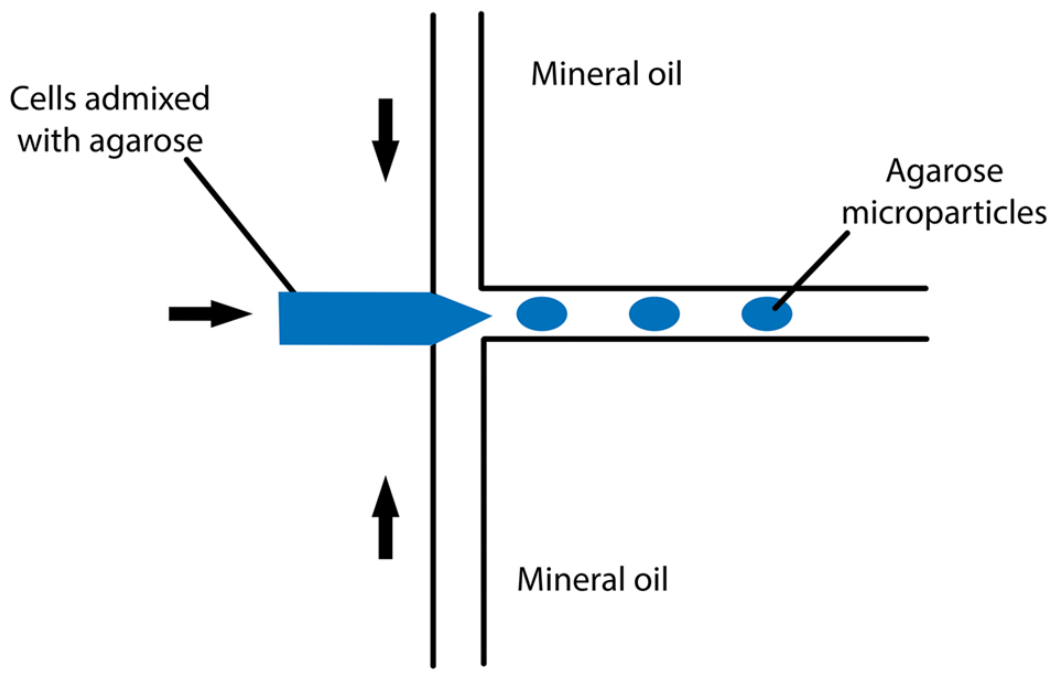

Figure 4: Schematic diagram of microfluidic device to produce bacteria with agarose hydrogel. Figure adapted from (25).

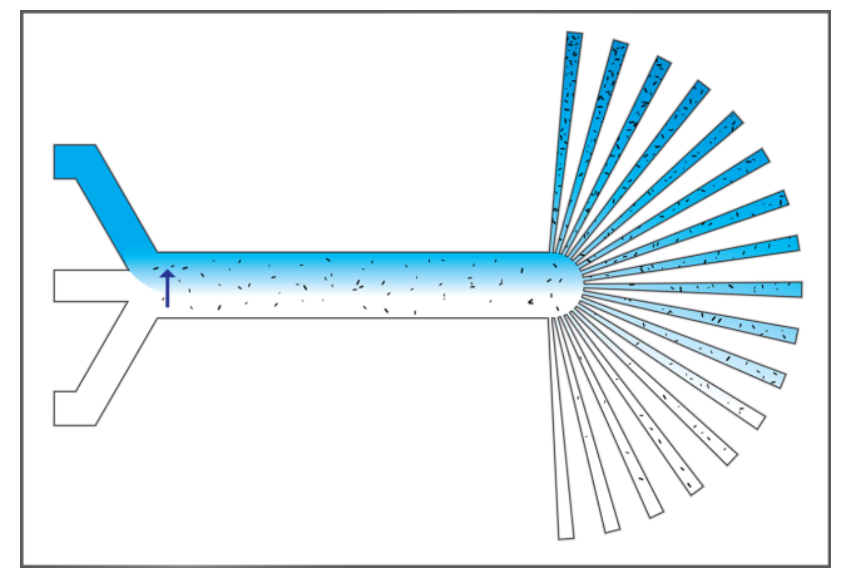

Figure 5: Microfluidic device to increase the concentration of bacteria by chemotaxis in different channels. Figure adapted from (27). 
Interaction of $E$. Coli with blood leading to coagulation: Once hydrogel beads containing $E$. Coli, are formed, blood mixed with calcium chloride are flown through one inlet of the Y-device shown in fig.3, while hydrogel beads could be infused through the other inlet. The size of the hydrogel beads has to be larger than the gap between the pillars in the channel. Therefore, these beads will get stuck in the triangular pillars. Blood will continue to flow through these porous hydrogels and interacts with the clusters of E. Coli. According to our proposed mechanism E. coli clusters will induce clot formation due to quorum acting phenomenon. To verify the proposed mechanism, output blood is examined. First, the output sample could be scrutinized for presence of clots.

If clots are found, test for factor $12 /$ factor 3 in plasma blood sample by various techniques like Prothombin Test (PT), activated partial thromboplastin time (aPTT), Thrombin time TT, chromogenic coagulation factor assays, etc could be performed (26). If the serum level of factor $12 / 3$ is found to be depleted or absent, we can conclude that $E$. coli-induced quorum acting follows similar pathway for intravascular coagulation which is analogous to intrinsic/extrinsic coagulation pathway. In another possible outcome, factor 12 or factor 3 may be present but still clotting occurs.

In that case, scrutinization for each coagulation factor individually could be performed along with the identification of factor which starts the process and propagates clot formation. Here we can infer that E. coli- induced quorum acting bypasses factor 12 or factor 3 and follows a pathway that is different from intrinsic or extrinsic pathway of coagulation respectively.

\section{Research direction for objective 2}

To establish that the bacteria e.g. E.coli clusters induce clot formation, a method to controllably increase/decrease the number of $E$. Coli confined in the hydrogel droplets has to be employed. Since a priori the quorum acting threshold number of E. Coli, is not known, we need to use the device shown in fig. 5 to gradually increase/decrease the number of $E$. Coli present in the droplets to identify the threshold number of E.coli required to induce coagulation.

For obtaining different numbers of bacteria at different outputs, a microfluidic device (Fig. 5) which is normally used to probe the chemotactic behaviour of bacteria could be used $(27,28)$. To encapsulate $E$. Coli in hydrogel droplets E.coli are segregated in required numbers by using a microfluidic device to confine E. Coli using its chemotaxis behavioural properties. The device contains three inlets (left side of Fig. 5).
Chemo-effector solutions are introduced through the upper inlet, buffer through the lower. A concentration gradient develops perpendicular to the direction of flow (shown by blue arrow in fig. 5).

A third, narrower inlet between these two streams is used to inject E. coli cells. The cells encounter the evolving chemical gradient as they move downstream. The gradient changes along the length of the channel, but it remains constant at any given point. At the far end (right side of Fig. 5) each cell enters one of 17 outlets according to the direction and extent of its migration normal to the direction of flow. Thus, greater numbers of $E$. Coli cells are seen towards the higher concentration of chemo-effectors (upper outlets).

Depending on the concentration of bacteria in the solution highest number of bacteria on the upper extreme outlet could be obtained. Later, all bacteria from each individual outlet could be confine into a single hydrogel droplet. Several such droplets with a similar number of confined bacteria could be used to test the coagulation. The number of bacteria could be increased or decreased depending on whether coagulation has occurred or not.

\section{Determining the threshold number of bacteria for Quorum acting.}

Let us denote the number of bacteria in the 17th outlet as "n17". We could confine all those bacteria in hydrogel droplets and perform the required experiment. If coagulation occurs, then further confirmation is required if " $\mathrm{n} 17$ " is the threshold number for initiating coagulation by quorum acting. For this, the 16th outlet has to be considered and all the bacteria from it are confined in hydrogel droplets .i.e. "n16" in hydrogel droplets and perform experiment similarly. If coagulation occurs than it could be inferred that "n17" was not the threshold stimulus.

Similarly, 15 th outlet could also be chosen to check if " $\mathrm{n} 16$ " is the threshold stimulus. In case if "n 15 " does not induce coagulation, inference could be drawn that "n16" is the threshold number to induce coagulation by quorum acting. The smallest number that leads to coagulation will be the threshold for quorum acting.

Thus, at this point we could have hydrogel beads which encapsulate clusters of bacteria sufficient to induce coagulation by quorum acting. We can segregate those agarose beads, liquefy them by increasing temperature and employ differential light scattering or optical density methods to quantify number of bacteria. 


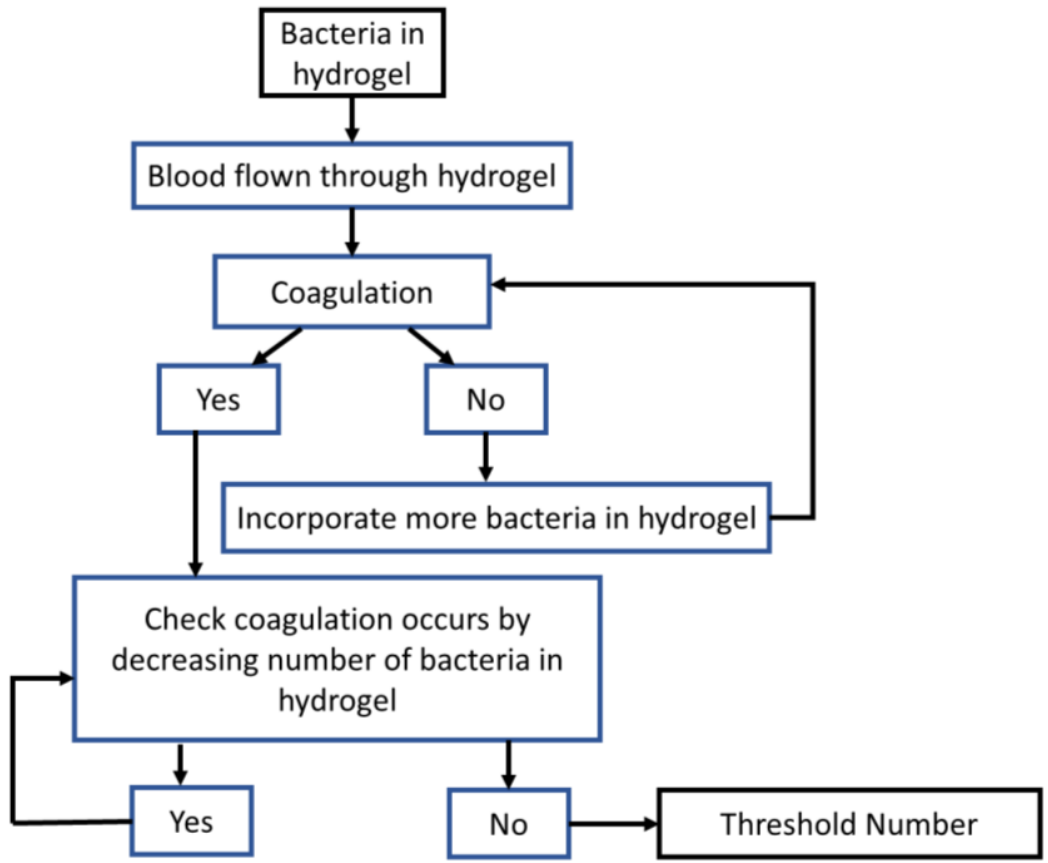

Figure 6 (A): Flow chart to determine the approximate threshold number of bacteria required for Quorum Acting

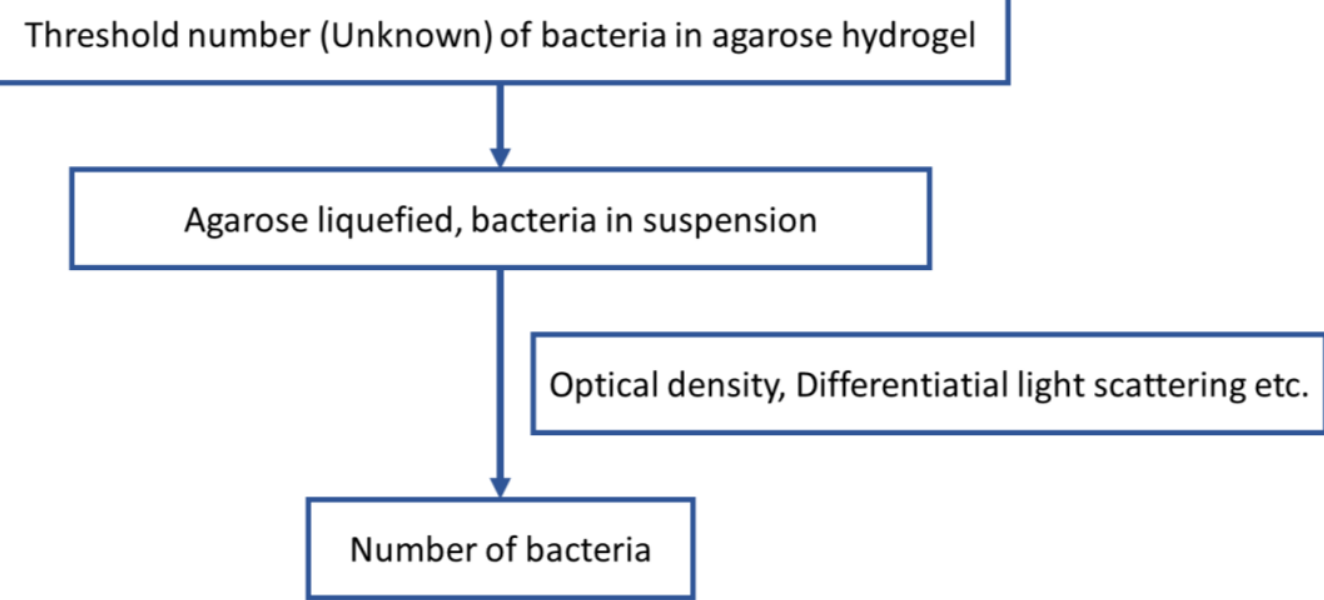

Figure 6 (B): Flow chart to determine the threshold number of bacteria required for Quorum Acting 
Device design and fabrication for confining $E$.coli and for determining the threshold stimuli:

The device for confining E. Coli comprises of modified "Y" shaped three separate inlets and array of outlet microchannels as shown in figure 5.

It could be designed with the help of Clewin software /AutoCAD mechanical drawing programme. PDMS could be used to construct the device with $20 \mathrm{~mm}$ central channel diameter.

The three separate inlets are used for chemoeffector, bacteria and buffer. The blue vertical arrow in fig. 5 shows the gradient of the chemoeffectors in the direction of highest concentration. The device for determining the threshold stimuli is shown in fig.3. All devices could be fabricated as described for objective 1 .

Production of agarose hydrogels containing bacteria: The similar protocol for bacterial encapsulation and clustering in agarose hydrogels as described in methodology for objective 1 could be used.

\section{Research direction for objective 3}

As mentioned earlier, sepsis and DIC occurs more frequently in patients admitted in critical care units with a past history of undergoing some invasive process like catheter insertion, sheath placement in femoral arteries, cannulation etc. which acts as portal of entry of bacteria into sterile bloodstream $(3,7,14$, 29). As they lie in bed $24 \times 7$ a condition called "venous stasis" is frequently observed in these patients $(3,7,14)$. Venous stasis is the sluggish flow of deoxygenated blood in large veins (Fig.7B)(3, 7, $14,29)$. Investigation is required to examine if quorum acting induced coagulation occurs more frequently in presence of venous stasis. For the above stated problem, a micro device which could mimic large venous space and passive sluggish blood flow as in large veins could be designed (Fig. 9).

Device design and fabrication: As shown in figure 8, the design comprises of oval interconnected reservoirs. These circular reservoirs represents large vein with inefficient valvular function. In the interconnected junctions micropillers, which will engage the hydrogel containing bacteria could be designed. The device fabrication protocol could follow the similar pattern as in objective 1 and 2 .

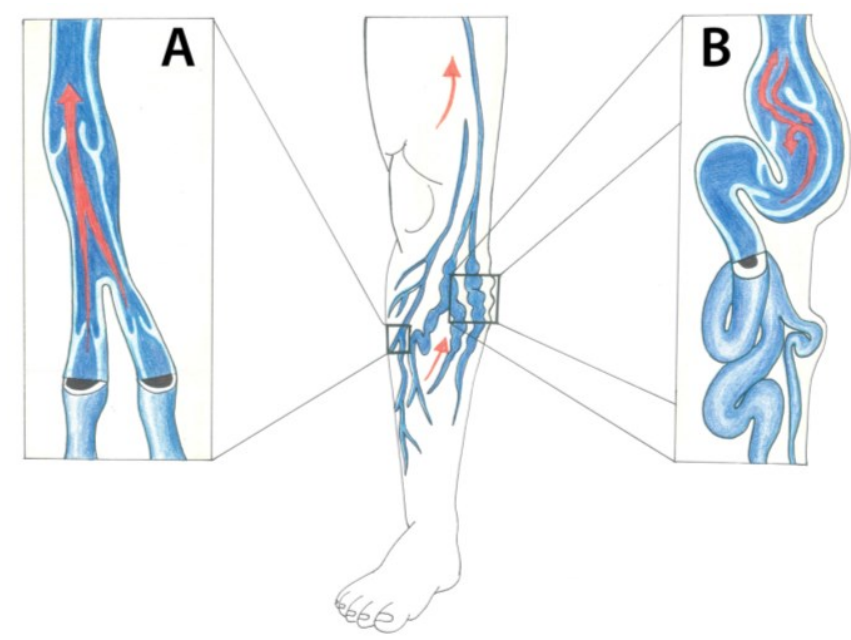

Figure 7 (A): Normal venous flow and (B) sluggish venous flow of blood (in venous stasis) Figure adapted from (45)

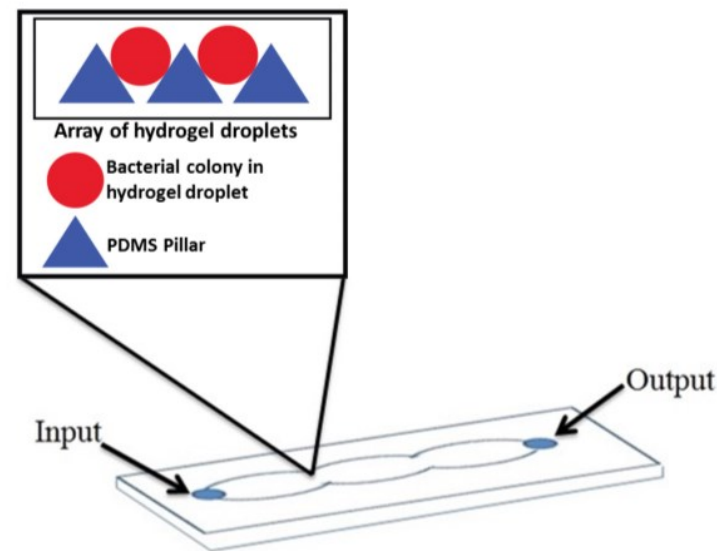

Figure 8: Design in the microfluidic device mimicking the tortuous flow of blood as in venous stasis cases 
Testing of the device using blood: Protocols described for objectives 1 and 2 may be followed to examine the sampled blood. A sluggish blood flow could be maintained with the help of syringe pump. Clotting time induced by E. coli could be noted and compared.

\section{Research direction for objective 4}

Sepsis and DIC are more frequently observed in patients admitted in critical care units with a history of uncontrolled diabetes mellitus (3, 7, 14, 30-32). A proper investigation is required to check if hyperglycaemic blood results more rapid quorum acting induced coagulation. To check the proposed mechanism, the same microdevice could be used as shown in figure 3. Instead of normal blood, hyperglycemic blood could be flown in this experiment. We could assume that E. Coli clusters will induce clot formation due to quorum acting phenomenon. Time taken for clot formation in hyperglycemic blood could be noted and compared with the results of normoglycemic blood.

Device design and fabrication: Same protocols as before could be followed.

Flow Testing of the device and experiments using blood: Same protocols as for objective-1 could be followed.

\section{Discussion}

In this proposed pathogenesis, we would like to examine (objectives 1 and 2) whether the coagulation cascade induced by $E$. Coli follows the intrinsic pathway/extrinsic pathway (i.e. starts from factor 12 /factor 3 ) or completely bypasses factor 12 /factor 3 and initiates coagulation using another factor further down the line (e.g., factor 11 , factor 9 , factor 10 , etc.in case of intrinsic pathway and factor 7 in case of extrinsic pathway). This could be verified by examining the clot induced in presence of $E$. Coli clusters and identifying the coagulation factors present in it. The clots could be examined by tests, such as, coagulation factor assay, chromogenic assay, prothombin time (PT), thrombin time (TT) and activated partial thromboplastin time (aPTT) etc $(3,7$, $14,26)$. If factor 12 or factor 3 is present in the clot, it would indicate that the $E$. coli-induced clot formation differs from the intrinsic or extrinsic pathway of coagulation and uses other downstream factors to initiate clot formation. Since bacterial cells can activate individual coagulation factors sporadically, we need to check the presence of individual coagulation factors (used and unused) and quantify the amount to establish the presence of a cascade. Presence of synchronized cascade in the experiment will establish that quorum acting mediates coagulation.

Sepsis and its complication DIC are more common in patients under critical care $(3,7,14)$. A condition called venous stasis is frequently observed in these patients $(3,7,14)$. Venous stasis (figure 7) is progressive decrease of venous blood flow. This condition results in swelling, hyperpigmentation and possible ulceration in the affected region $(3,7,14$, 29). Carefull investigation is to be carried out to examine if thrombosis and venous stasis are somehow related. More specifically, study should focus on whether quorum acting induced coagulation occurs more rapidly in blood which exhibits venous stasis (objective 3 ).
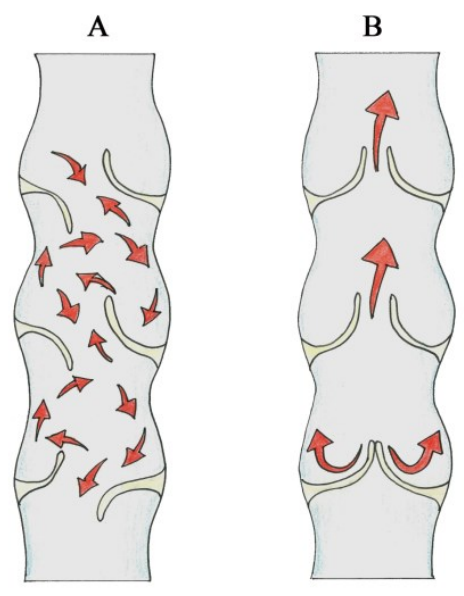

Figure 9 (A): Venous stasis (B) Normal venous flow. Figure adapted from (46)

Uncontrolled diabetes mellitus is a major risk factor for development of sepsis and its complication DIC in ICU/CCU patients. $(3,7,14,30,31)$. Diabetes is a lifelong metabolic condition that causes a person's blood sugar level to become too high $(3,7,14)$. Experiment to validate whether hyperglycemic blood provides a niche for more rapid activation of coagulation by quorum acting when compared to normoglycemic blood (objective 4 ) could be carried out to test the proposed mechanism.

Additionally, It is possible to mimic these clinical conditions in vitro using microfluidic device. Microfluidics involves controlled manipulation of very small volume of fluids in channels with dimensions of the order of tens to hundreds of micrometers (22-24). High surface-to volume ratio and low Reynolds number make viscous forces more dominant than inertial forces inside microfluidic devices $(22,23)$. Low Reynolds number results in laminar flow inside the microchannel (23) which mimics capillary hemodynamics. Some other factors which support the use of microfluidics for studying hemodynamics are: 1) Dimension of microchannels $(\approx$ 50-80 $\mu \mathrm{m})$ is similar to capillary, venules and arterioles. 2) Physiological flow rates $(1 \mathrm{~mm} / \mathrm{sec})$ are possible in microchannels during hemodynamic experiments. 3) Conditions like hyperglycemia, venous stasis and condition like spreading of bacteria in blood (haematogenous spread) can be simulated 
accurately with the help of microfluidics.4) Specialized protein in the microchannel could be flown to form a layer in the wall or coat the surface of the microchannel by endothelial cells (using process called RGD) which will mimic physiologically, the outermost layer of lumen or innermost layer of blood vessel made of endothelial cells (tunica intima). 5) Technique like micro contact printing technology, photolithography etc. could be used to coat biomaterial of interest (fibrinogen, collagen patches or tissue factor etc.) in microchannels.

Many clinical studies on sepsis and DIC are currently underway in universities and teaching hospitals. Much of the research is focused on the pharmacokinetics of drugs targeted towards

sepsis/DIC (33) and sepsis biomarkers (34-36). Kastrupet al in 2008 (17) showed that presence of bacteria, such as, Bacillus cereus and Bacillus anthracis can induce coagulation by quorum acting. Recently, a microfluidic system was used to show coagulation of blood in presence of tissue factors (TF) (37-42). Microcontact printing was used by Okorie et al (40) to generate a surface of collagen and TF to support both platelet aggregation and coagulationin a flow chamber. Ismagilov and colleagues (43) generated arrays of TF patches using photolithographic techniques to study the role of diffusion in coagulation. Collagen and TF were patterned inside microchannels to generate distinct regions of thrombosis by Colace et al (42). However, there are currently no known work that uses a microfluidic approach to probe sepsis and DIC.

\section{Conclusion}

Hemodynamic experiments mimicking arterial and venous blood flow could be studied using microfluidic technique. Apart from possible investigation of DIC, other diseased condition like hyperglycaemic blood of diabetics, venous stasis etc. can also be efficiently probed by this technique as discussed in the proposed mechanism.

Conflict of Interest: The authors declare no potential conflicts of interest with respect to the research, authorship, and/or publication of this article.

Acknowledgement: Author Contributions: SD, AB: Concept,Design, Invivo and Invitro studies, writing of article, Editing.

Ethical issues: All Authors declare that Originality of research/article etc... and ethical approval of research, and responsibilities of research against local ethics commission are under the Authors responsibilities. The study was conducted due to defined rules by the Local Ethics Commission guidelines and audits.

\section{References}

1. Hall J, Schmidt G, Wood L. Principles of critical care: McGraw-Hill Prof Med/Tech; 2005.

2. Torio CM, Andrews RM. National inpatient hospital costs: the most expensive conditions by payer, 2011. 2013

3. Longo D, Fauci A, Kasper D, Hauser S. Harrison's Principles of Internal Medicine 18th edition: McGraw-Hill Professional; 2011

4. Kottke-Marchant K, Pathologists CoA. An Algorithmic Approach to Hemostasis Testing: College of American Pathologists; 2008 .

5. Hall JE. Guyton and Hall textbook of medical physiology: Elsevier Health Sciences; 2010.

6. Levy M, Fink M, Marshall J, Abraham E, Angus D, Cook $\mathrm{D}$, et al., editors. for the international sepsis definitions conference (2003) 2001 SCCM: ESICM/ACCP/ATS/SIS International Sepsis Definitions Conference. Intensive Care Med 29: 530-538.

7. Cecil RLF, Goldman L, Schafer AI. Goldman's Cecil Medicine, Expert Consult Premium Edition--Enhanced Online Features and Print, Single Volume, 24: Goldman's Cecil Medicine: Elsevier Health Sciences; 2012.

8. Bone RC, Balk RA, Cerra FB, Dellinger RP, Fein AM, Knaus WA, et al. Definitions for sepsis and organ failure and guidelines for the use of innovative therapies in sepsis. The ACCP/SCCM Consensus Conference Committee. American College of Chest Physicians/Society of Critical Care Medicine. Chest Journal. 1992;101(6):1644-55.

9. Hunter P. Sepsis under siege. EMBO reports. 2006;7(7):667-9.

10. Semeraro N, Ammollo CT, Semeraro F, Colucci M. Sepsis-associated disseminated intravascular coagulation and thromboembolic disease. Mediterranean journal of hematology and infectious diseases. 2010;2(3).

11. Dunn DL. Gram-negative bacterial sepsis and sepsis syndrome. The Surgical clinics of North America. 1994;74(3):621-35

12. Angus DC, Linde-Zwirble WT, Lidicker J, Clermont G, Carcillo J, Pinsky MR. Epidemiology of severe sepsis in the United States: analysis of incidence, outcome, and associated costs of care. Critical care medicine. 2001;29(7):1303-10.

13. Linde-Zwirble WT, Angus DC. Severe sepsis epidemiology: sampling, selection, and society. Critical Care. 2004;8(4):222.

14. Colledge N, Walker B, Ralston S. DavidsonÆs principles $\&$ Practice of Medicine. 21st. Edition Churchill Livingston. 2010 .

15. Simon F, Scheuerle A, Soell A, Groeger M, McCook O, Radermacher $\mathrm{P}$, et al. 30th International Symposium on Intensive Care and Emergency Medicine. Critical Care. 2010;14(1):P1.

16. Beekmann S, Diekema D, Chapin K, Doern G. Effects of rapid detection of bloodstream infections on length of hospitalization and hospital charges. Journal of clinical microbiology. 2003;41(7):3119-25.

17. Kastrup CJ, Boedicker JQ, Pomerantsev AP, Moayeri M, Bian Y, Pompano RR, et al. Spatial localization of bacteria controls coagulation of human blood by'quorum acting'. Nature chemical biology. 2008;4(12):742-50. 
18. Shen F, Pompano RR, Kastrup CJ, Ismagilov RF. Confinement regulates complex biochemical networks: initiation of blood clotting by "diffusion acting". Biophysical journal. 2009;97(8):2137-45.

19. Miller MB, Bassler BL. Quorum sensing in bacteria. Annual Reviews in Microbiology. 2001;55(1):165-99.

20. Judy JW. Microelectromechanical systems (MEMS) fabrication, design and applications. Smart materials and Structures. 2001;10(6):1115.

21. Thompson LF, Willson CG, Bowden MJ. Introduction to microlithography. 1983.

22. Anna SL, Bontoux N, Stone HA. Formation of dispersions using "flow focusing" in microchannels. Applied physics letters. 2003;82(3):364-6.

23. Teh S-Y, Lin R, Hung L-H, Lee AP. Droplet microfluidics. Lab on a Chip. 2008;8(2):198-220.

24. Takeuchi S, Garstecki P, Weibel DB, Whitesides GM. An Axisymmetric Flow - Focusing Microfluidic Device. Advanced materials. 2005;17(8):1067-72.

25. Eun Y-J, Utada AS, Copeland MF, Takeuchi S, Weibel DB. Encapsulating bacteria in agarose microparticles using microfluidics for high-throughput cell analysis and isolation. ACS chemical biology. 2010;6(3):260-6.

26. Bates SM, Weitz JI. Coagulation assays. Circulation. 2005;112(4):e53-e60.

27. Wessel AK, Hmelo L, Parsek MR, Whiteley M. Going local: technologies for exploring bacterial microenvironments. Nature Reviews Microbiology. 2013;11(5):337-48.

28. Mao H, Cremer PS, Manson MD. A sensitive, versatile microfluidic assay for bacterial chemotaxis. Proceedings of the National Academy of Sciences. 2003;100(9):5449-54

29. Heit JA, Rooke TW, Silverstein MD, Mohr DN, Lohse $\mathrm{CM}$, Petterson TM, et al. Trends in the incidence of venous stasis syndrome and venous ulcer: a 25 -year populationbased study. Journal of Vascular Surgery. 2001;33(5):1022-7.

30. Rayfield EJ, Ault MJ, Keusch GT, Brothers MJ, Nechemias C, Smith H. Infection and diabetes: the case for glucose control. The American journal of medicine. 1982;72(3):439-50.

31. Joshi N, Caputo GM, Weitekamp MR, Karchmer A Infections in patients with diabetes mellitus. New England Journal of Medicine. 1999;341(25):1906-12.

32. Butler SO, Btaiche IF, Alaniz C. Relationship between hyperglycemia and infection in critically ill patients. Pharmacotherapy: The Journal of Human Pharmacology and Drug Therapy. 2005;25(7):963-76.

33. Sadaka F, O'Brien J, Migneron M, Stortz J, Vanston A, Taylor RW. Activated protein $\mathrm{C}$ in septic shock: a propensity-matched analysis. Crit Care. 2011;15(2):R89.
34. Gradwohl-Matis I, Dünser MW. On sepsis, troponin and vasopressin: the bitter truth. Critical Care. 2013;17(5):1002.

35. Skibsted S, Bhasin MK, Aird WC, Shapiro NI. Bench-tobedside review: future novel diagnostics for sepsis-a systems biology approach. Crit Care. 2013;17(5):231.

36. Jyothi P, Basavaraj MC, Basavaraj PV. Bacteriological profile of neonatal septicemia and antibiotic susceptibility pattern of the isolates. Journal of natural science, biology, and medicine. 2013;4(2):306

37. Mackman N. Role of tissue factor in hemostasis, thrombosis, and vascular development. Arteriosclerosis, thrombosis, and vascular biology. 2004;24(6):1015-22.

38. Kastrup CJ, Runyon MK, Lucchetta EM, Price JM, Ismagilov RF. Using chemistry and microfluidics to understand the spatial dynamics of complex biological networks. Accounts of chemical research. 2008;41(4):54958

39. Runyon MK, Johnson-Kerner BL, Kastrup CJ, Van Ha TG, Ismagilov RF. Propagation of blood clotting in the complex biochemical network of hemostasis is described by a simple mechanism. Journal of the American Chemical Society. 2007;129(22):7014-5.

40. Okorie UM, Denney WS, Chatterjee MS, Neeves KB, Diamond SL. Determination of surface tissue factor thresholds that trigger coagulation at venous and arterial shear rates: amplification of $100 \mathrm{fM}$ circulating tissue factor requires flow. Blood. 2008;111(7):3507-13.

41. Kastrup CJ, Shen F, Runyon MK, Ismagilov RF. Characterization of the threshold response of initiation of blood clotting to stimulus patch size. Biophysical journal. 2007;93(8):2969-77.

42. Colace TV, Jobson J, Diamond SL. Relipidated tissue factor linked to collagen surfaces potentiates platelet adhesion and fibrin formation in a microfluidic model of vessel injury. Bioconjugate chemistry. 2011;22(10):21049.

43. Shen F, Kastrup CJ, Ismagilov RF. Using microfluidics to understand the effect of spatial distribution of tissue factor on blood coagulation. Thrombosis research. 2008;122:S27S30.

44. http://www.elveflow.com/microfluidic-tutorials/softlithography-reviews-and tutorials/introduction-in-softlithography/introduction-about-soft-lithography-andpolymer-molding-for-microfluidic/ Last assessed on 28-112016

45. http://www.veinguide.com/blog/288/varicose-veins-veinvalves-and-venous-insufficiency-Last accessed on 28-112016

46. http://www.veinsofhouston.com/venous-insufficiency Last accessed on 28-11-2016

Copyright (C) 2016 The Author(s); This is an open-access article distributed under the terms of the Creative Commons Attribution License (http://creativecommons.org/licenses/by/4.0), which permits unrestricted use, distribution, and reproduction in any medium, provided the original work is properly cited. All Rights reserved by international journal of Medical Science and Discovery. 